\title{
Two-dimensional stress measurement of a micromachined piezoresistive structure with micro-Raman spectroscopy
}

\author{
J. Qian, T.-X. Yu, Y.-P. Zhao
}

\begin{abstract}
This paper describes the stress characterization of a cantilever structure of a piezoresistive microflowmeter using micro-Raman spectroscopy. In order to obtain the relationship between the stress and the shift of Raman frequency, the mechanical stress in the structure was assumed to be uniaxial according to the applied loading and the boundary conditions. Also, the two-dimensional stress distribution of the structure was simulated using a finite element tool (ABAQUS v6.2). The experimental results agree well to those predicted by the finite element simulation. It is concluded that micro-Raman spectroscopy is an accurate, non-destructive technique for measuring Microelectromechanical systems (MEMS) local stress with micrometer spatial resolution.
\end{abstract}

\section{1}

\section{Introduction}

Microelectromechanical systems (MEMS) are evolving at a rapid rate in a wide variety of applications [1]. Micromachined piezoresistive structures are among the most popular approaches to microsensors. In these micromachined devices, accurate characterization of mechanical stress is crucial for the successful design, fabrication and operation [2].

The mechanical stress field can be obtained by finite element modeling (FEM). However, there has been few experimental verification of FEM used in structure design. Several experimental methods can deliver information on mechanical stress in MEMS devices. The most important techniques are X-ray diffraction, transmission electron

Received: 14 July 2003 / Accepted: 15 March 2004

J. Qian, Y.-P. Zhao ( $₫)$

State Key Laboratory of Nonlinear Mechanics (LNM), Institute of Mechanics, Chinese Academy of Sciences, Beijing 100080, China

e-mail: yzhao@lnm.imech.ac.cn

T.-X. Yu

Department of Mechanical Engineering,

Hong Kong University of Science and Technology,

Clear Water Bay, Kowloon, Hong Kong

The supports from the Key Project from the Chinese Academy of Sciences (No. KJCX2-SW-L2), projects from the National Natural Science Foundation of China (Nos. 10225209, 50131160739 and 90305020), the National "973" project (No. G1999033103), and the Joint Laboratory of Microsystems between the Institute of Mechanics (CAS) and HKUST are gratefully acknowledged. microscopy (TEM), and micro-Raman spectroscopy; all of these methods have advantages and disadvantages. As devices become smaller, spatial resolution becomes a more and more important criterion to decide the usefulness of a technique for stress measurement in MEMS. For X-ray diffraction, the size of the probing spot is in the range of $1 \mathrm{~mm}^{2}$. The TEM method reaches spatial resolutions of a few nanometers, but the main disadvantages of it are the destructive sample preparation and the extensive modeling required to extract the stress information from the measurement.

Micro-Raman spectroscopy reaches neither the stress sensitivity of X-ray diffraction nor the spatial resolution of TEM, but it has been recognized to be a promising technique for rapid and non-destructive stress measurement with micrometer spatial resolution $[3,4]$. As an example of such verification, we considered the applied bending stress in a micromachined piezoresistive cantilever structure, to show that micro-Raman spectroscopy can be used as an effective measuring technique to determine local and induced stress in MEMS devices.

\section{2}

\section{Sample preparation}

The silicon structure of interest is shown in Fig. 1, which is composed of two different plates with geometries of $(400 \times 250) \mu \mathrm{m}^{2}$ and $(1300 \times 1150) \mu \mathrm{m}^{2}$. This suspension structure with a piezoresistor at its root has been applied to a gas microflowmeter [5]. The structure is $25 \mu \mathrm{m}$ thick. The top surface shown in Fig. 1 is the $(100)$ plane and the axis of the cantilever is oriented parallel to the [110] direction of single crystal silicon.

\section{1}

\section{Fabrication process}

The complete fabrication was performed at Institute of Microelectronics of Peking University and its process is illustrated in Fig. 2. An n-type (100) silicon wafer was used as substrate. After growing a $100 \mathrm{~nm}$ thickness thermal oxide layer, Mask 1 was used to pattern windows, through which boron for p-type piezoresistors was implanted (Fig. 2a). Subsequently, wet oxidation was carried out at $1100^{\circ} \mathrm{C}$ for 10 minutes in order to drive boron to desired depth and grow oxide for the next diffusion step. The resistance of the piezoresistors was about $3.5 \mathrm{k} \Omega$. Next step is $\mathrm{P}+$ diffusion; Mask 2 was used to pattern $\mathrm{P}+$ areas that would be ohmic contact between p-type piezoresistors and metal lines. A reactive ion etcher (RIE) flow was followed to remove oxide film (Fig. 2b). Next, the second diffusion 


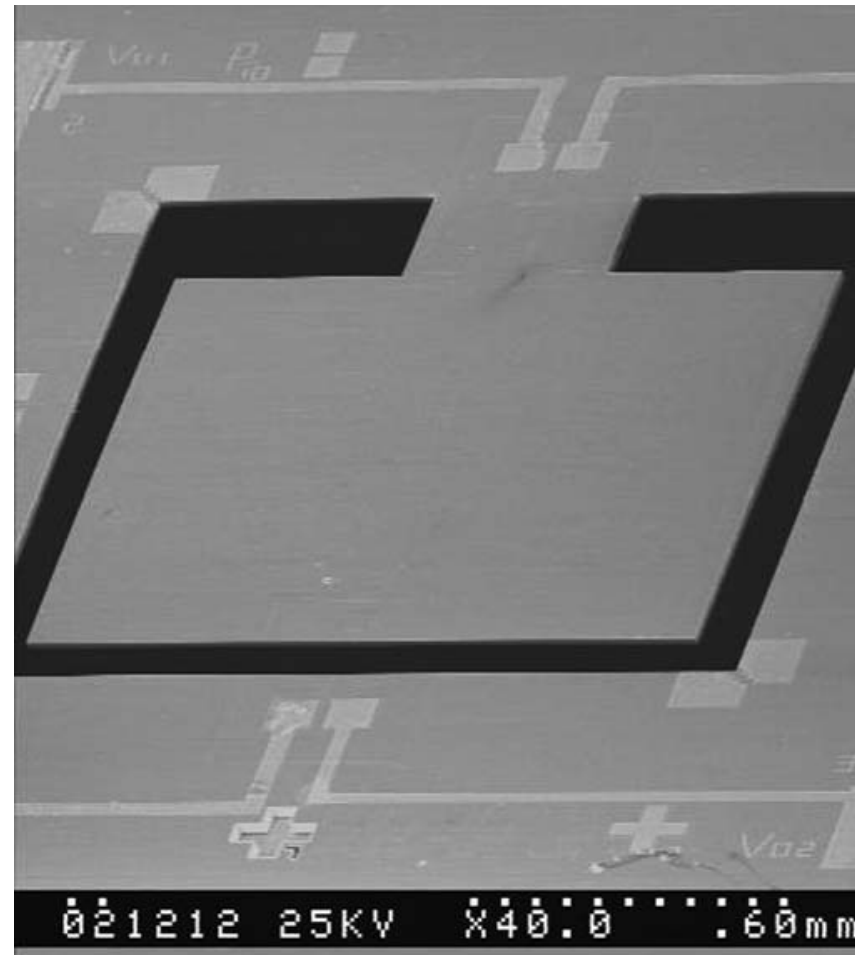

Fig. 1. Scanning electron micrograph (SEM) of the piezoresistive cantilever plate

was defined by Mask 3 and $\mathrm{N}+$ region was formed, making the substrate equipotential (Fig. 2c).

Oxide on both surfaces of the wafer was stripped. A new oxide/nitride bi-layer was deposited by low-pressure chemical vapor deposition (LPCVD) in order to create a mask for the following anisotropic wet chemical etching. The thicknesses of the oxide and nitride were 400 and $160 \mathrm{~nm}$, respectively. The backside oxide/nitride was patterned using Mask 4, and a membrane was fabricated by $\mathrm{KOH}$ solution (Fig. 2d). By choosing etching time and parameters of the etchant, desired size and thickness of the membrane, 1500 and $25 \mu \mathrm{m}$ for these devices, were achieved.

Subsequently, the nitride layer was removed. Mask 5 was used to open contact windows on the left oxide layer. Aluminum was deposited by sputtering and patterned by Mask 6 , followed by an annealing process at $450^{\circ} \mathrm{C}$ for $30 \mathrm{~min}$ (Fig. 2e).

Finally, Mask 7 for etching holes in the membrane was used. The silicon membrane was etched by an inductive coupled plasma (ICP) system (Fig. 2f). ICP systems have excellent performance on geometry shaping, especially for such a structure with low depth about $25 \mu \mathrm{m}$. However, slight under-cut occurred at the fringe of the etched patterns. This phenomenon is called notching [6], which affected the shape of our microstructures in some sort and introduced a little error between the designed model and the practical devices.

After fabrication, an $80 \mu \mathrm{m}$ diameter and $1 \mathrm{~mm}$ length wire was placed on the top surface near the end of the suspension structure. The wire was aligned parallel to the [-110] direction in order to avoid torsion effects during the following loading process. The whole structure with

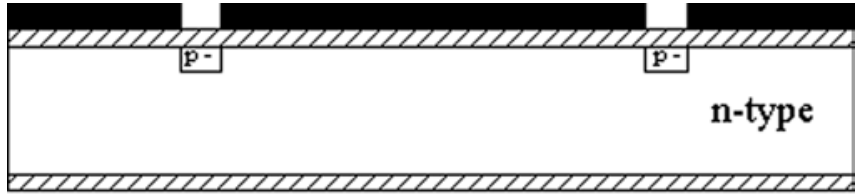

(a)

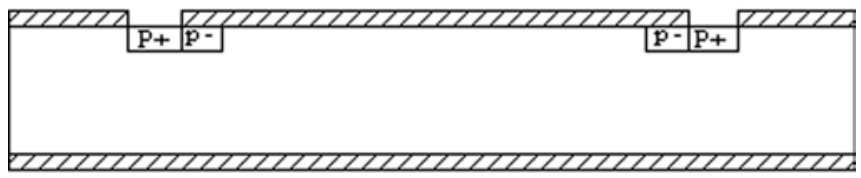

(b)

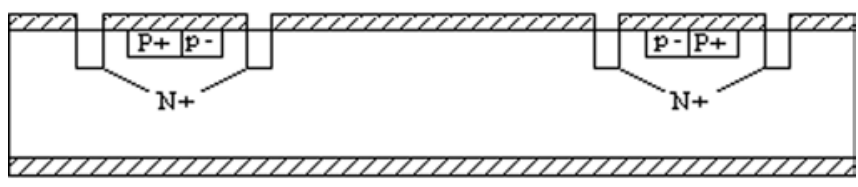

(c)

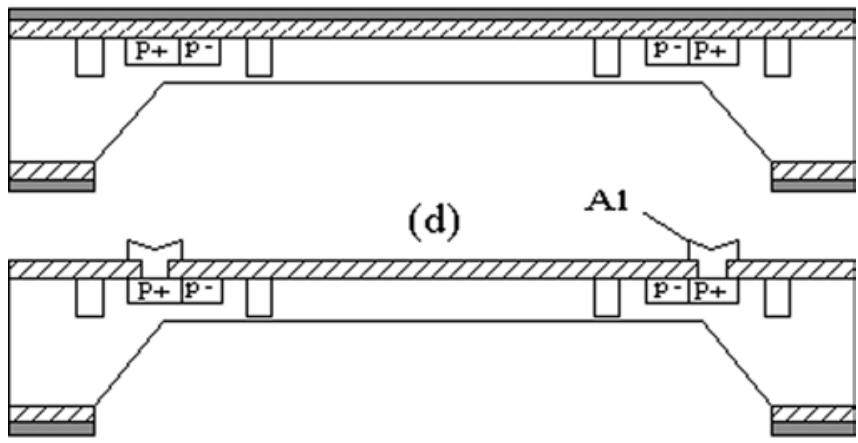

(e)
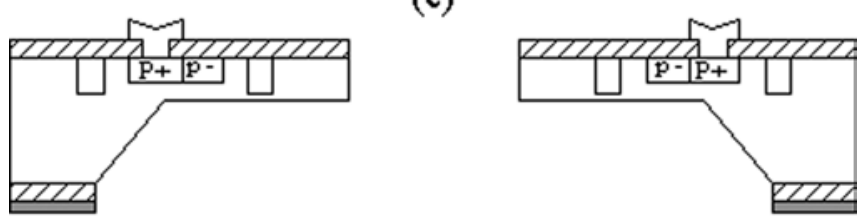

(f)

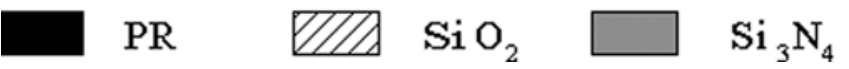

Fig. 2a-f. The fabrication process of the peizoresistive cantilever structure. a boron implantation for p-piezoresistors, $\mathbf{b}$ boron diffusion for $\mathrm{P}+$ regions, $\mathrm{c}$ phosphorus diffusion for $\mathrm{N}+$ regions, d $\mathrm{KOH}$ etching for silicon membrane, e deposition of aluminum, f drilling holes by ICP system

the wire on it was inserted between two parallel glassslides, as shown in Fig. 3. After the two glass-slides were clamped together and closed to each other, the deflection of the loading position, i.e. the position where the wire lay, was measured to be $74.1 \mu \mathrm{m}$. The error in this optical measurement is estimated to be about $1 \mu \mathrm{m}$. The distance from the loading position to the end edge was measured to be about $200 \mu \mathrm{m}$.

\section{2}

\section{Analytical model (Euler beam theory)}

The induced stress in the structure can be studied using an analytical model, based on the theory of materials mechanics [7]. Here the cantilever plate is considered to be an Euler beam with two different inertia moments, $I_{1}$ and 


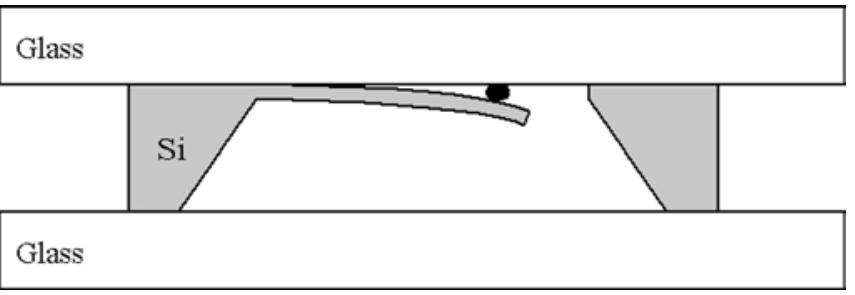

Fig. 3. The loading process in samples preparation

$I_{2}$, as shown in Fig. 4. This implies the following assumptions: (i) the structure is homogeneous and perfectly elastic; (ii) the structure deforms in pure bending; and (iii) Poisson contraction or expansion is not constrained. It should be remarked that generally the third assumption is valid for narrow beams. We accept it because the Poisson's ratio of silicon for the orientation under consideration is very small (only 0.064 in [8]).

As illustrated in Fig. 4, the method of superposition can be used to determine the force $P$ acting on point $C$. The Young's modulus of the material is $E$, and the lengths of the parts $A B$ and $B C$ are $L_{1}$ and $L_{2}$, respectively. First, we imagine that point $B$ of the structure is held rigidly so that it neither deflects nor rotates, then the deflection $\delta_{C}$ of point $\mathrm{C}$ will be:

$\delta_{C}=\frac{P L_{2}^{3}}{3 E I_{2}}$.

However, the part $A B$ of the structure also behaves like a cantilever and contributes to the deflection of point $C$. The deflection $\delta_{B}$ and rotation angle $\theta_{B}$ of point $B$ are:

$\delta_{B}=\frac{P L_{1}^{3}}{3 E I_{1}}+\frac{\left(P L_{2}\right) L_{1}^{2}}{2 E I_{1}}, \quad \theta_{B}=\frac{P L_{1}^{2}}{2 E I_{1}}+\frac{\left(P L_{2}\right) L_{1}}{E I_{1}}$.

The deflection $\delta_{B}$ and rotation angle $\theta_{B}$ make an additional contribution to the deflection of point $C$. Therefore, the total deflection of point $C$ is:
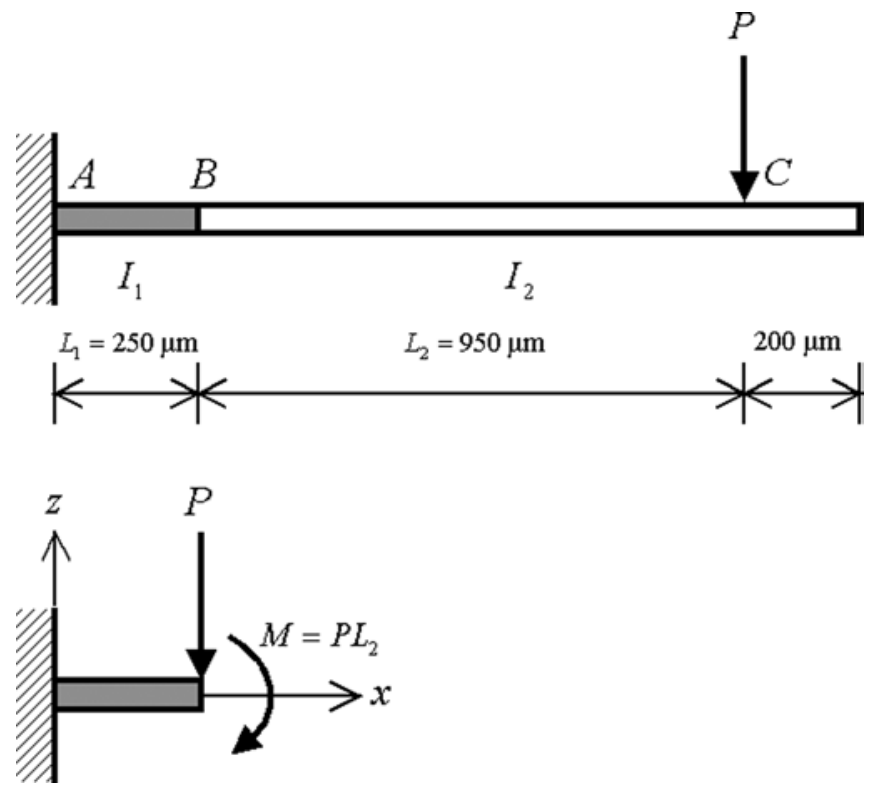

Fig. 4. Cantilever structure with two different inertia moments
$\delta=\delta_{C}+\delta_{B}+\theta_{B} \times L_{2}$.

Recall that the deflection $\delta$ has been measured to be $74.1 \mu \mathrm{m}$. By Eqs. (1-3), the load $P$ can be calculated to be $17.22 \mathrm{mN}$. The value of the Young's modulus used in the above calculation is $169 \mathrm{GPa}$, which is the value along the [110] direction in the (001) plane of silicon. Therefore, the spatial variation of $\sigma_{x x}$ in the part $A B$ is given by

$\sigma_{x x}=\frac{P\left(L_{1}+L_{2}-x\right) \cdot z}{I_{1}} \quad\left(0 \leq x \leq L_{1},-t / 2 \leq z \leq t / 2\right)$.

This model predicts that the maximum tensile stress, which is a quantity of interest to designers, occurs at the root of the cantilever structure. However, the assumptions of Euler beam are expected to be invalid in some region due to the structure width as well as the stress concentration at the corners. In order to take these effects into consideration, numerical analysis was performed using the finite element model.

\section{3}

\section{Finite element model (FEM)}

The stress in the sample was analyzed using a finite element tool-ABAQUS v6.2. Two-dimensional finite element model was created using the 8-node shell element and totally 2136 elements were employed in the mesh(as shown in Fig. 5). Single crystal silicon was modeled as an isotropic material because we expected that the main stress component is along the [110] direction for the loaded sample, and we set its Young's modulus and Poisson's ratio to be $169 \mathrm{GPa}$ and 0.064 , respectively, which are theoretical values of the [110] direction within silicon

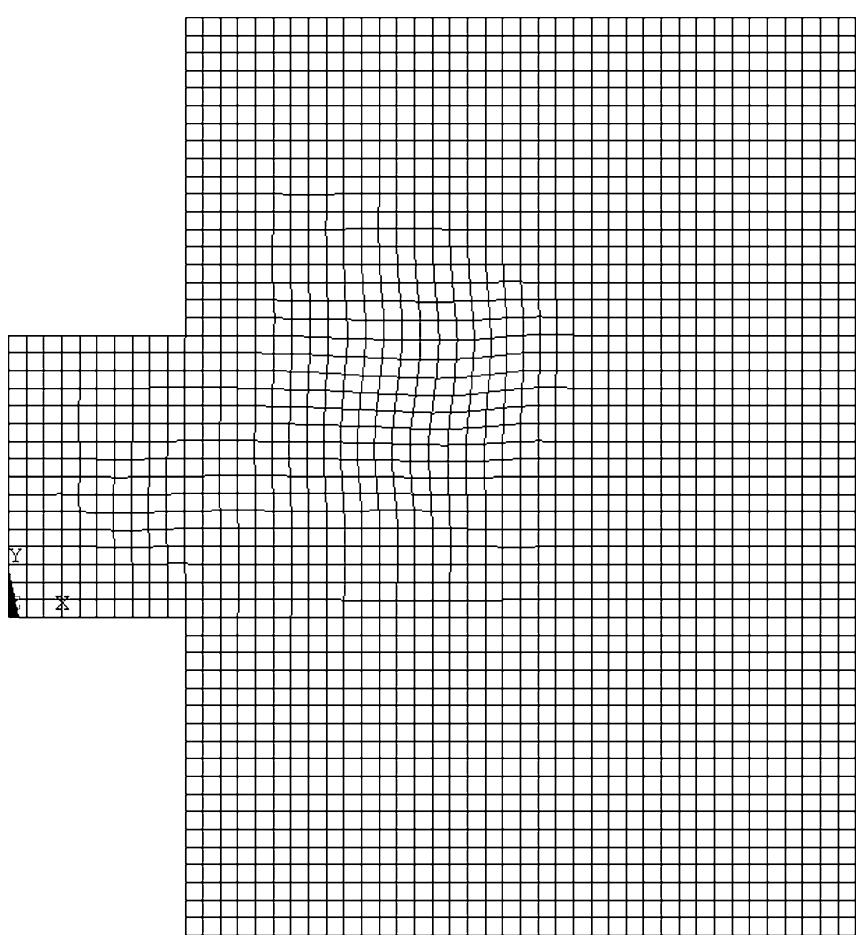

Fig. 5. The shell element mesh used to model the mechanical stress of the sample 
(1 00 ) plane [8]. Fixed boundary conditions were applied to the root of the cantilever structure, and displacement of $74.1 \mu \mathrm{m}$ was applied to the loading position.

In the region near the support, the maximum difference of 70-80 MPa in $\sigma_{x x}$ occurs through the structure width.

The maximum tensile stress occurs at the corners of point $B$, indicating a modest stress concentration. The FEM results also indicate that the normal stress along $x$ axis is much larger than the other stress components.

\section{3}

\section{Micro-Raman spectroscopy}

\section{1}

\section{Theory}

One of the first papers theoretically addressing the effect of strain on Raman frequency was written by Ganesan et al. [9]. The relation between Raman shift and mechanical strain for diamond-structured crystals can be obtained by solving the following "secular equation":

$$
\left|\begin{array}{ccc}
p \varepsilon_{11}+q\left(\varepsilon_{22}+\varepsilon_{33}\right)-\lambda & 2 r \varepsilon_{12} & 2 r \varepsilon_{13} \\
2 r \varepsilon_{12} & p \varepsilon_{22}+q\left(\varepsilon_{11}+\varepsilon_{33}\right)-\lambda & 2 r \varepsilon_{23} \\
2 r \varepsilon_{13} & 2 r \varepsilon_{23} & p \varepsilon_{33}+q\left(\varepsilon_{11}+\varepsilon_{22}\right)-\lambda
\end{array}\right|=0
$$
reference system. Next, the strain tensor is rotated to the reference system. This results in:

$\varepsilon_{33}=\varepsilon_{33}^{\prime}$,

$\varepsilon_{12}=\frac{\varepsilon_{11}^{\prime}-\varepsilon_{22}^{\prime}}{2}$,

$\varepsilon_{13}=\varepsilon_{23}=0$,

where $\varepsilon_{i j}$ are the strain components in the reference systhree eigenvalues can easily be obtained. These three eigenvalues and corresponding eigenvectors reflect three Raman-active optical photon modes of silicon: two transverse and one longitudinal. In the absence of any stress and for backscattering from the $\left(\begin{array}{lll}0 & 0 & 1\end{array}\right)$ plane, the trans- where $p, q, r$ are the photon deformation potentials [10], which are material constants, and $\varepsilon_{i j}$ are the strain tensor components. The eigenvalues, $\lambda$, give the relation between the shift of Raman frequency and the strain. If the Raman frequency under study is $\omega$ and its stress-free value is $\omega_{0}$ ( $520 \mathrm{~cm}^{-1}$ for silicon), the shift of Raman frequency induced by the strain, $\Delta \omega$, is given by:

$\Delta \omega=\omega-\omega_{0} \approx \frac{\lambda}{2 \omega_{0}}$.

According to Eqs (5) and (6), all the six strain tensor components influence the position of the Raman peak, but in experiment only one peak is observed. It is impossible to obtain the six unknown variables from one experimental value. So some prior assumptions concerning the magnitude of the different strain tensor components are required.

In the simplest approximation, it has been assumed that only the normal stress along the [110] direction, $\sigma_{11}^{\prime}$, is nonzero in our sample. In the presence of this uniaxial stress assumption, the secular equation has to be solved. Because the secular equation is given in the reference system $x=[100], y=[010]$, and $z=[001]$, we have to calculate the strain components in this system. Firstly, the strain components, $\varepsilon_{i j}^{\prime}$, in the sample system $x^{\prime}=[110]$, $y^{\prime}=[-110]$, and $z^{\prime}=[001]$ are calculated using Hooke's law:

$\varepsilon_{11}^{\prime}=\left(\frac{S_{11}+S_{12}}{2}+\frac{S_{44}}{4}\right) \sigma_{11}^{\prime}$,

$\varepsilon_{22}^{\prime}=\left(\frac{S_{11}+S_{12}}{2}-\frac{S_{44}}{4}\right) \sigma_{11}^{\prime}$,

$\varepsilon_{33}^{\prime}=S_{12} \sigma_{11}^{\prime}$,

$\varepsilon_{12}^{\prime}=\varepsilon_{13}^{\prime}=\varepsilon_{23}^{\prime}=0$, where $S_{i j}$ are the elements of the compliance tensor in the

$\varepsilon_{11}=\varepsilon_{22}=\frac{\varepsilon_{11}^{\prime}+\varepsilon_{22}^{\prime}}{2}$, tem. Substituting these strain components into the secular equation results in a non-diagonal matrix, from which the

verse modes are polarized along [100] and [010], respectively, and the longitudinal mode is polarized along [001]. When the silicon lattice is strained, the frequencies of the photon modes can change from the stress-free value. For our specific case of a uniaxial stress applied along the [110] direction, De Wolf et al. [11] have calculated that only the third photon mode, i.e. the longitudinal mode, will be visible. So:

$\Delta \omega_{3}=\frac{\lambda_{3}}{2 \omega_{0}}=\frac{\left[q\left(S_{11}+S_{12}\right)+p S_{12}\right]}{2 \omega_{0}} \sigma_{11}^{\prime}$.

The experimental values of the parameters of single crystal silicon in Eq. (9) are given by references $[8,12]$ :

$S_{11}=7.67 \times 10^{-12} \mathrm{~Pa}^{-1}$,

$S_{12}=-2.13 \times 10^{-12} \mathrm{~Pa}^{-1}$,

$p / \omega_{0}^{2}=-1.85 \pm 0.06$,

$q / \omega_{0}^{2}=-2.31 \pm 0.06$.

Thus, the linear relation between the shift of Raman frequency and the uniaxial stress along the [110] direction is calculated to be:

$\sigma_{11}^{\prime}[\mathrm{MPa}]=435 \Delta \omega\left[\mathrm{cm}^{-1}\right]$.

By Eq. (11), the uniaxial stress in our sample can be derived from the measured shift of Raman frequency. An increase of Raman frequency indicates tensile stress, and a decrease of Raman frequency indicates compressive stress. In the following experiments, we measured the stress map of the narrow part of the sample and compared the results with those predicted by analytical and finite element models. 


\section{2}

\section{Raman measurements}

The measurements of the induced stress in the cantilever structure were performed using a Renishaw system (model RM3000) in backscattering mode. The laser was incident

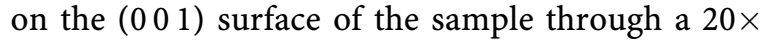

microscope objective, and the scattered light was collected using the same objective. The laser used was a He-Ne laser at $632.8 \mathrm{~nm}$, and the laser power on sample was limited to $2.5 \mathrm{~mW}$ to minimize sample heating.

First, we used a piece of unstrained silicon as reference. In this case, we did reference measurements with this unstrained silicon before and after the sample scan to see whether a frequency change had occurred. However, external factors, such as the instrument stability and the room temperature, affected the position of the Raman frequency, so it was difficult for us to obtain the exact frequency change induced by the strain. For the purpose of calibration, the plasma lines of the laser were utilized, because their frequency is determined by the laser tube and will not change during the investigation. As indicated in Fig. 6, we can clearly see the silicon Raman peak and some very thin plasma lines in the spectra.

Experimental scans were performed on the bottom surface of the narrow part of the sample (as shown in Fig. 7). We didn't map the top surface because the ion implantation process could affect the Raman frequency (Fig. 8). The scan region was $(400 \times 250) \mu \mathrm{m}^{2}$ and the thickness of the sample was $25 \mu \mathrm{m}$. In order to obtain the accurate position the peaks in Raman spectrum, the measured silicon Raman peaks were fitted with a Lorentzfunction, and the plasma lines of the laser tube were fitted using a Gauss-function.

\section{4}

\section{Results and discussion}

The result of this $11 \times 11$ two-dimensional scan over the entire investigated region, giving the uniaxial compressive stress map at the bottom surface, is given in Fig. 9. At the top surface, the stress map is expected to be reversed. We also measured the stress at the top surface along line 2 in

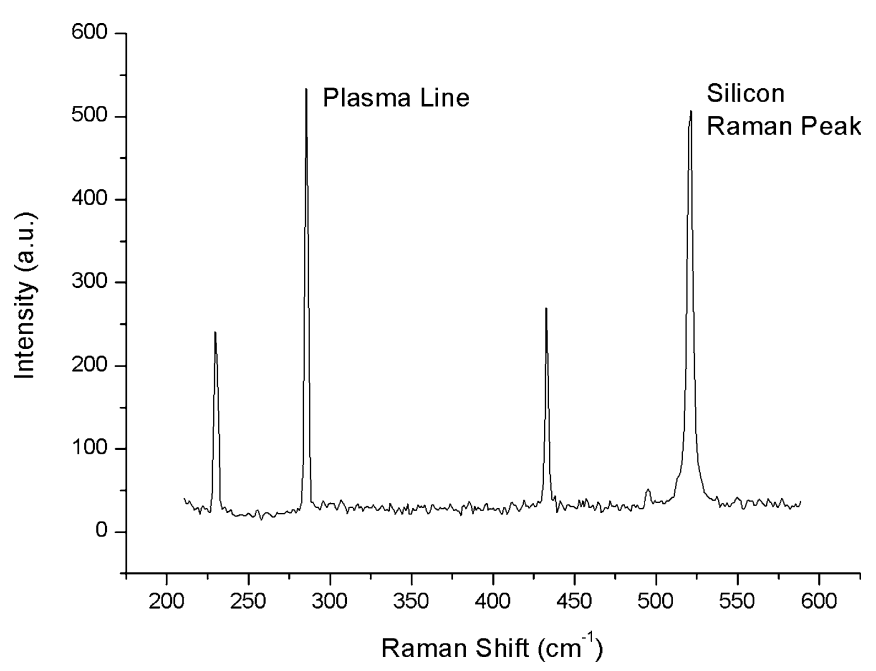

Fig. 6. Raman spectrum of silicon with plasma lines

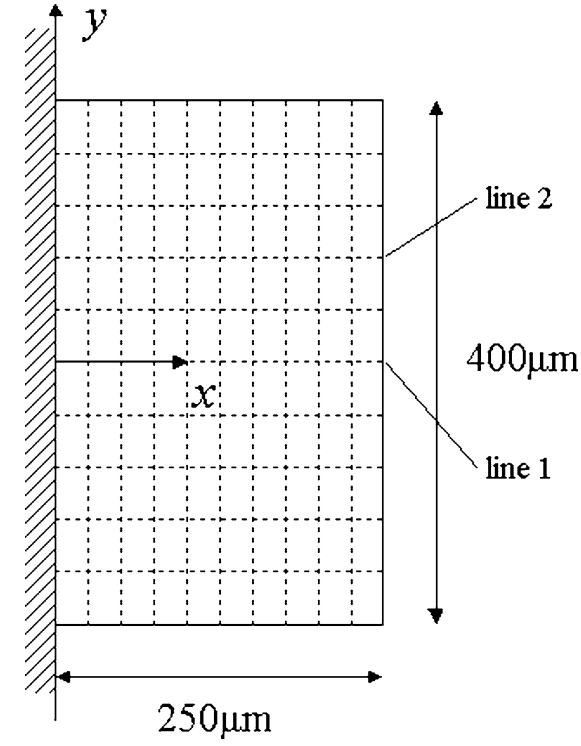

101

Fig. 7. The scan grid of the investigated region

Fig. 7, since this line didn't go through the ion diffusion regions. It is clear that the experimental results of the top stress scan and bottom stress scan have the same absolute value and opposite sign, as shown in Fig. 10.

Next, we considered the mechanical stress along line 1 in Fig. 7, where piezoresistors are generally located.

Figure 11 gives the stress profiles from the analytical model, finite element model and experimental measurement. The experimental results were found to be in good agreement with the prediction of FEM along the entire line, the stress matches to $6 \%$. Also, it is clear that the Euler beam model overestimates the stress. Our calculation indicates that the analytical solution given by Euler beam model overestimates the stress by $10-30 \%$ in the scan region. This error occurs because the assumptions of Euler beam model are violated in the investigated region.

The penetration depth $d_{p}$ (10\% intensity left) of the $632.8 \mathrm{~nm} \mathrm{He}-\mathrm{Ne}$ laser in single crystal silicon can be obtained by [13]:

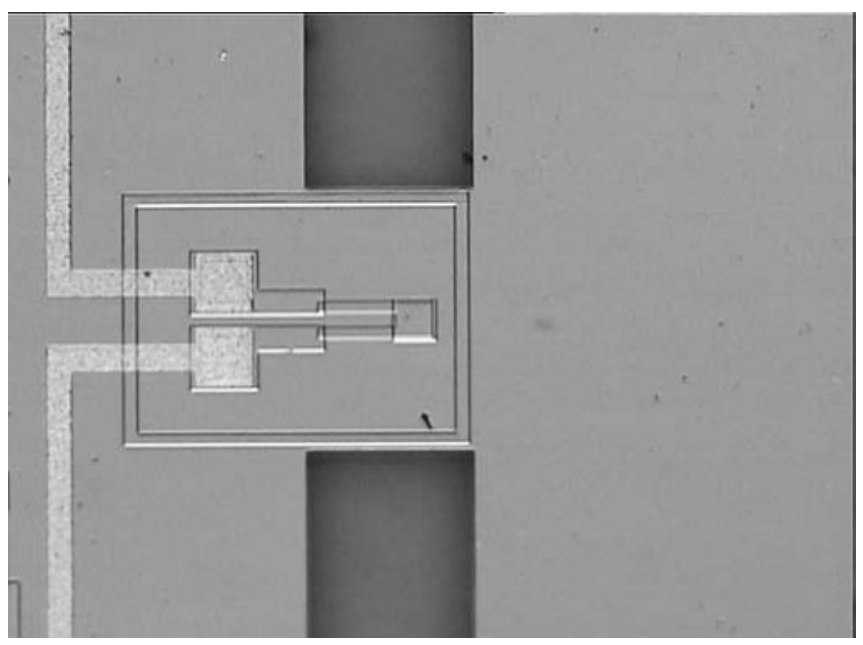

Fig. 8. The photograph of the piezoresistive region of the structure 


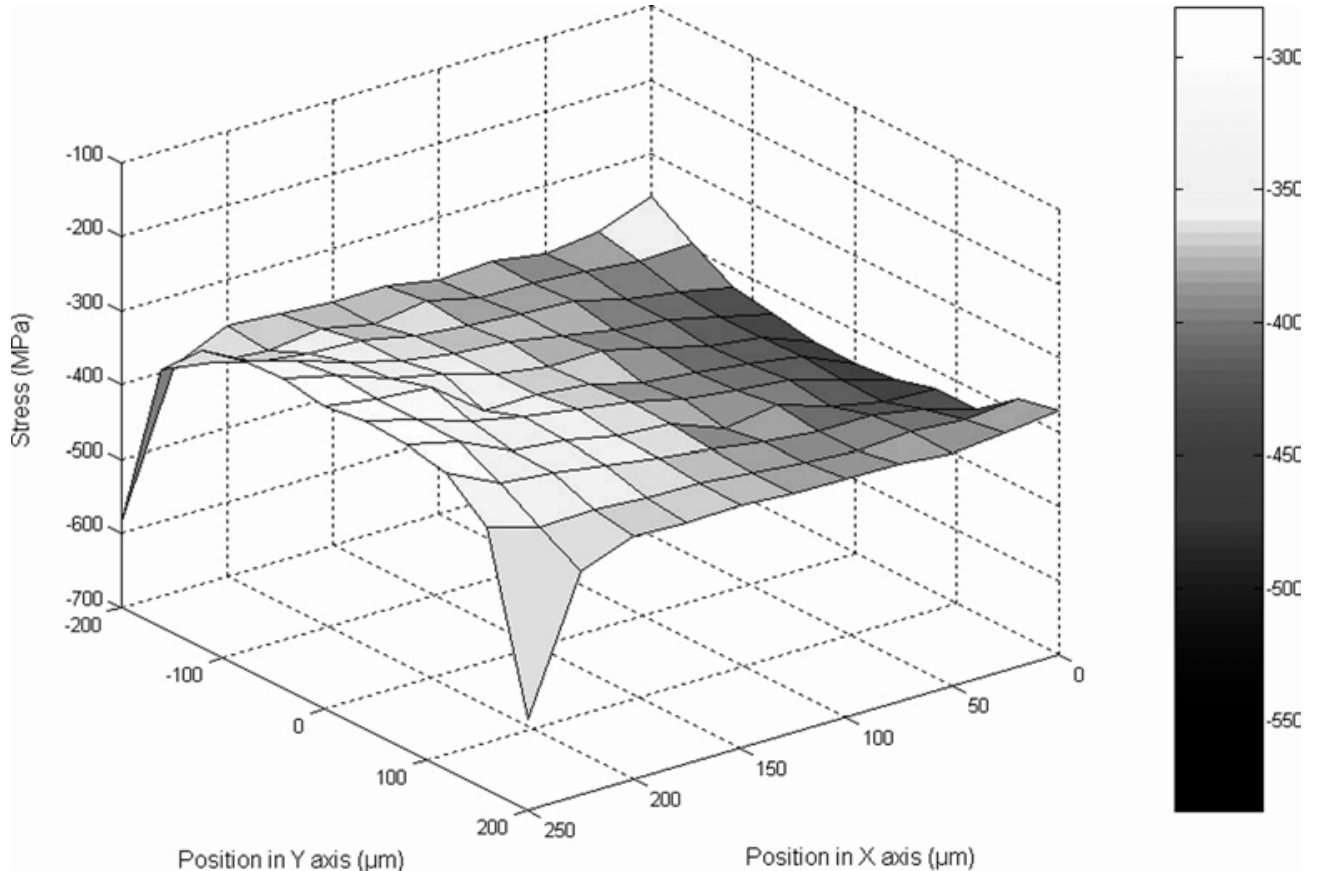

Fig. 9. Two-dimensional stress profile in the investigated region measured with microRaman spectroscopy $d_{p}=\frac{-\ln 0.1}{2 \alpha}=\frac{2.3}{2 \alpha}$,

where $\alpha$ is the absorption coefficient of silicon. For the $632.8 \mathrm{~nm}(1.96 \mathrm{eV}) \mathrm{He}-\mathrm{Ne}$ laser, $\alpha$ is $3.94 \times 10^{3} \mathrm{~cm}^{-1}$ [14] and the penetration depth is calculated to be $2.919 \mu \mathrm{m}$. This indicates that the Raman scattering light in our measurement contains not only the stress information at the top (or bottom) surface, but also that of the inner materials. This can partially explain the fact that most experimental results are lower than those predicted by FEM.

\section{5}

\section{Conclusions}

We have measured the induced and local stress in a bulkmicromachined piezoresistive structure with micro-

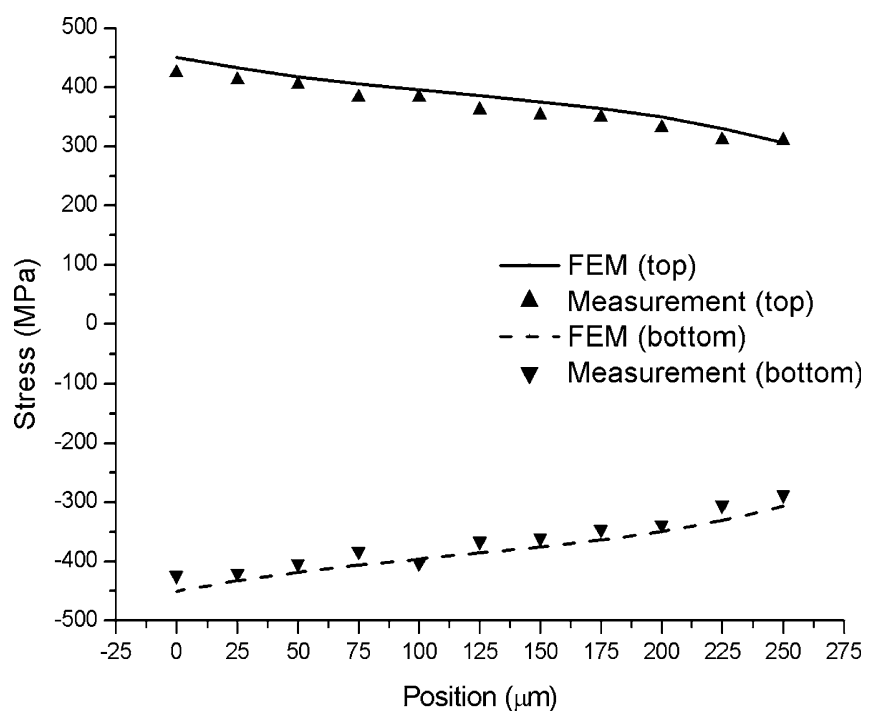

Fig. 10. The comparison between the top measurement and bottom measurement along a scan line $(y=80 \mu \mathrm{m})$
Raman spectroscopy, and modeled the structure using Euler beam model and finite element model. The following conclusions can be made:

1. Micro-Raman spectroscopy can offer an accurate, nondestructive measurement for local stress in MEMS device. In our measurement, the experimental results agree well with those predicted by the finite element model (the stress matches to 6\%).

2. Analytical model based on Euler beam theory significantly overestimate the stress to $10-30 \%$ for our structure. This is caused by the fact that the structure width and the stress concentration violate the assumptions of Euler beam theory.

3. The spatial resolution is less good due to the long wavelength of $\mathrm{He}-\mathrm{Ne}$ laser. The laser penetrates deep in

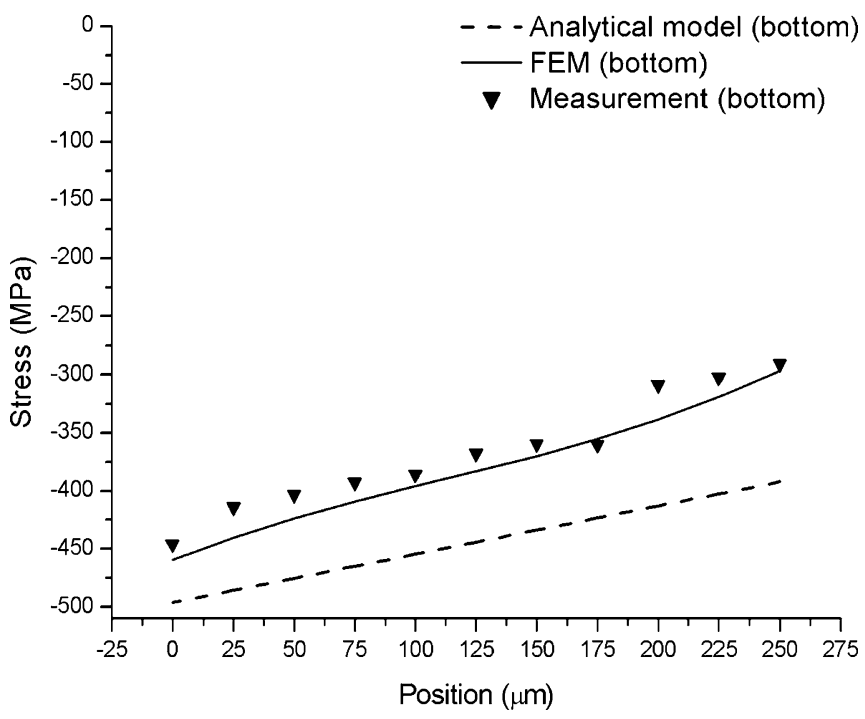

Fig. 11. The stress profiles from the analytical model, finite element model and experimental measurement along a scan line $(y=0)$ 
the silicon, so the measured results contain the stress information of the materials beneath the surface.

There are several possible sources inducing the error between the measurement and FEM: (1) the imperfect assumption of uniaxial stress in measurement, (2) improper identification of boundary conditions in FEM, (3) the use of material properties of silicon for boron/phosphorus implanted region, (4) imprecise dimension of etched structures, and (5) sample heating induced by laser.

\section{References}

1. Madou M (1997) Fundamentals of microfabrication. CRC Press

2. Senturia SD (2001) Microsystem design, Kluwer Academic Publishers

3. De Wolf I; Maes HE (1998) Mechanical stress measurements using micro-Raman spectroscopy. Microsys Technol 5: 13-17

4. De Wolf I; Chen J; van Spengen WM (2001) The investigation of microsystems using Raman spectroscopy. Optics Lasers Eng 36: 213-223

5. Wang XB; Qian J; Zhang DC (2004) Fabrication, testing and mechanical analysis of bulk-micromachined flowmeters. Acta Mech Sinica 20(2): 152-158

6. Jung CO; Chi KK; Hwang BG; Moon JT; Lee MY; Lee JG (1999) Advanced plasma technology in microelectronics. Thin Solid Films 341(1-2): 112-119
7. Timoshenko SP; Gere JM (1973) Mechanics of Materials. Van Nostrand Reinhold Company Ltd., London

8. Brantley WA (1973) Calculated elastic constants for stress problems associated with semiconductor devices. J Appl Phys 44: 534-535

9. Ganesan S; Maradudin A; Oitmaa J (1970) A lattice theory of morphic effects in crystals of the diamond structure. Ann Phys 56: 556-594

10. De Wolf I (1996) Micro-Raman spectroscopy to study local mechanical stress in silicon integrated circuits. Semiconductor Sci Technol 11: 139-154

11. De Wolf I; Maes HE; Jones SK (1996) Stress measurements in silicon devices through Raman spectroscopy: bridging the gap between theory and experiment. J Appl Phys 79(9): 71487156

12. Anastassakis E; Cantarere A; Cardona M (1990) Piezo-Raman measurements and anharmonic parameters in silicon and diamond. Phys Rev B 41(11): 7529-7535

13. Paillard V; Puech P; Sirvin R; Hamma S; Cabarrocas PR (2001) Measurement of the in-depth stress profile in hydrogenated microcrystalline silicon thin films using Raman spectrometry. J Appl Phys 90(7): 3276-3279

14. Aspnes DE; Studna AA (1983) Dielectric functions and optical parameters of $\mathrm{Si}, \mathrm{Ge}, \mathrm{GaP}, \mathrm{GaAs}, \mathrm{GaSb}$, InP, InAs and InSb from 1.5 to $6.0 \mathrm{eV}$. Phys Rev B 27: 985-1008 